\title{
Marked length rigidity for symmetric spaces
}

\author{
Françoise Dal'Bo and Inkang Kim ${ }^{1}$
}

\begin{abstract}
We give conditions under which a homomorphism between two Zariski dense subgroups of connected semisimple Lie groups $G$ and $G^{\prime}$ without compact factors and with trivial center can be extended to a continuous isomorphism between $G$ and $G^{\prime}$. In particular we prove the marked length rigidity and the marked translation vector rigidity. This last result was motivated by a Margulis's question.
\end{abstract}

Mathematics Subject Classification (2000). 22E40, 22F30.

Keywords. Symmetric spaces, length spectrum.

\section{Introduction}

Let $G, G^{\prime}$ be connected semisimple Lie groups without compact factors and with trivial center. The motivation of this paper is to give conditions under which a homomorphism between two Zariski dense subgroups of $G$ and $G^{\prime}$ can be extended to a continuous isomorphism between $G$ and $G^{\prime}$. Much study of lattices has been done, yet the study of general co-infinite volume groups is relatively less carried out. Fix a closed Weyl chamber $\mathcal{A}^{+}$included in the Lie algebra of $G$. The translation vector $v(g)$ of $g \in G$, is, by definition, the unique $a \in \mathcal{A}^{+}$such that $e^{a}$ is conjugate to the hyperbolic part of the Jordan decomposition of $g$ (see section 1 ). The Euclidean norm of $v(g)$ is denoted $\ell(g)$ and is called the length of $g$. If $X$ is a symmetric space associated to $G$, one has: $\ell(g)=\operatorname{Inf}_{x \in X} d(x, g(x))$. In the particular case where $G=\operatorname{PSL}(n, \mathbb{R})$ and $\mathcal{A}^{+}$is the set of diagonal matrices $\operatorname{diag}\left(a_{1}, \cdots, a_{n}\right)$ with $a_{1} \geq \cdots \geq a_{n}$, one has: $v(g)=\operatorname{diag}\left(\log \left|\lambda_{1}\right|, \cdots \log \left|\lambda_{n}\right|\right)$ where $\lambda_{i}$ is the $i^{\text {th }}$ complex eigenvalue of $g$. Let $\Gamma \subset G$, the limit cone, $\mathcal{L}(\Gamma)$, associated to $\Gamma$ is, by definition, the smallest closed cone in $\mathcal{A}^{+}$containing all $v(\gamma)$ for $\gamma \in \Gamma$. An important result due to Y. Benoist [1] says that the interior of $\mathcal{L}(\Gamma)$ is not empty, if $\Gamma$ is a Zariski dense group. The originality of this paper is to explore this property to obtain strong rigidity results in a short and elementary way.

Let us give the main results.

\footnotetext{
${ }^{1}$ Partially supported by the KOSEF interdisciplinary grant 1999-2-101-5.
} 
Theorem A. Let $\Gamma \subset G, \Gamma^{\prime} \subset G^{\prime}$ be Zariski dense subgroups. If $\varphi$ is a surjective homomorphism between $\Gamma$ and $\Gamma^{\prime}$ such that $\ell(\gamma)=\ell(\varphi(\gamma))$ for any $\gamma \in \Gamma$ then $\varphi$ can be extended to a continuous isomorphism between $G$ and $G^{\prime}$.

Following the way of A. Parreau [15], we give applications of Theorem A to the space of representations of an abstract group into $G$.

Theorem A is already known for symmetric spaces of rank 1 ([4], [11]) and their products ([12]). For simple Lie groups it is shown in ([6]). Along this line, Besson, Courtois, Gallot and Hamenstädt ([2], [9]) showed that, if $M$ is a negatively curved locally symmetric compact manifold and $N$ is an arbitrary negatively curved manifold which has the same marked length spectrum with $M$, then they are isometric. Actually it is conjectured that two negatively curved compact manifolds with the same marked length spectrum are isometric. This conjecture is proved in dimension 2 ([14]).

The following theorem gives a positive answer to a Margulis's question raised during the rigidity conference at Paris in June 1998.

Theorem B. Suppose $G=G^{\prime}$ and rank $G \geq 2$. Let $\Gamma, \Gamma^{\prime}$ be Zariski dense subgroups of $G$. If $\varphi$ is a surjective homomorphism between $\Gamma$ and $\Gamma^{\prime}$ such that for all $\gamma \in \Gamma$ there exists $k(\gamma) \in \mathbb{R}^{*}$ such that $v(\varphi(\gamma))=k(\gamma) v(\gamma)$, then $\varphi$ can be extended to a continuous automorphism of $G$.

We first study the simple case where $G$ and $G^{\prime}$ are simple. Using a criterion of conjugacy proved in [6] we give a family of conditions (including conditions of Theorems A and B) under which a surjective homomorphism between Zariski dense subgroups can be extended.

\section{Benoist's theorem for limit cone}

An element $g$ of a real reductive connected linear group can be uniquely written $g=e h u$ where $e$ si elliptic (all the eigenvalues have modulus 1 ), $u$ is unipotent ( $u$ Id is nilpotent), $h$ is hyperbolic (all the eigenvalues are real positive), and all three commute. This decomposition is called the Jordan decomposition of $g$. If $G=\mathrm{KAN}$ is any Iwasawa decomposition of a connected semisimple Lie group $G$, then $e$ is conjugate to an element in $K, h$ is conjugate to an element in $A$ and $u$ is conjugate to an element in $N([1],[7])$. Fix a closed Weyl chamber $\mathcal{A}^{+}$in the Lie algebra of $G$, there exists a unique $a \in \mathcal{A}^{+}$, called the translation vector of $g$ and denoted $v(g)$, such that $h$ is conjugate to $e^{a}$. Geometrically, if $X$ is a symmetric space associated to $G$, then $\|v(g)\|=\ell(g)$ where $\ell(g)=\operatorname{Inf}_{x \in X} d(x, g(x))$ (see [15] for an interpretation of $v(g)$ ). Let $\Gamma$ be a subgroup of $G$, one defines the limit cone of $\Gamma$, denoted $\mathcal{L}(\Gamma)$, as the smallest closed cone in $\mathcal{A}^{+}$containing $v(\Gamma)$. If $G=\operatorname{PSL}(2, \mathbb{R}) \times \operatorname{PSL}(2, \mathbb{R})$ and $\mathcal{A}^{+}=\left\{\left(r_{1} M, r_{2} M\right) / r_{1}, r_{2} \in \mathbb{R}^{+}\right\}$where $M=\left(\begin{array}{rr}1 & 0 \\ 0 & -1\end{array}\right)$, then $\mathcal{L}(\Gamma)$ is the closure 
of $\left\{\left(r \ell\left(\gamma_{1}\right) M, r \ell\left(\gamma_{2}\right) M\right) / r \in \mathbb{R}^{+},\left(\gamma_{1}, \gamma_{2}\right) \in \Gamma\right\}$ where $\ell\left(\gamma_{i}\right)=0$ if $\gamma_{i}$ is elliptic or parabolic and $\ell\left(\gamma_{i}\right)>0$ is the displacement of $\gamma_{i}$ if $\gamma_{i}$ is hyperbolic. The following result, due to Y. Benoist, plays a key role in this paper.

Theorem 1.1 [1]. If $\Gamma$ is a Zariski dense subgroup of $G$ then $\mathcal{L}(\Gamma)$ is convex and has nonempty interior.

In the particular case where $\Gamma$ is a Zariski dense subgroup of $\operatorname{PSL}(2, \mathbb{R}) \times$ $\operatorname{PSL}(2, \mathbb{R})$ associated to the diagonal action of two isomorphic Fuchsian groups $\Gamma_{1} \stackrel{\varphi}{\longrightarrow} \Gamma_{2}$, this theorem says that $\left\{\frac{\overline{\ell\left(\gamma_{1}\right)}}{\ell\left(\varphi\left(\gamma_{1}\right)\right)}, \gamma_{1} \in \Gamma_{1}\right\}$ is an interval $[a, b] \subset$ $[0, \infty]$ with $a \neq b$. This property was already remarked in the context of rank 1 semisimple groups by M. Burger [4] (see also [5]).

\section{Rigidity results for simple groups}

In this section one supposes that $G$ and $G^{\prime}$ are connected, noncompact, simple Lie groups with trivial center. Let $\varphi: \Gamma \rightarrow \Gamma^{\prime}$ be a homomorphism between two subgroups of $G$ and $G^{\prime}$. One defines the graph group $\Gamma_{\varphi} \subset G \times G^{\prime}$ by $\Gamma_{\varphi}=$ $\{(\gamma, \varphi(\gamma)) / \gamma \in \Gamma\}$. The following result is proved in $[6]$.

Criterion of conjugacy 2.1 [6]. Let $\varphi$ be a surjective homomorphism between two Zariski dense subgroups $\Gamma, \Gamma^{\prime}$ included in connected non compact simple Lie groups, $G$ and $G^{\prime}$, with trivial center. The following properties are equivalent:

1) $\varphi$ can be extended to a continuous isomorphism between $G$ and $G^{\prime}$

2) $\Gamma_{\varphi}$ is not Zariski dense in $G \times G^{\prime}$.

This criterion is false if $G$ a $G^{\prime}$ are not simple. Take for example $G=\operatorname{PSL}(2, \mathbb{R})$ and $G^{\prime}=G \times G$. Denote $\mathcal{A}^{+}$the closed Weyl chamber of $G$ defined by $\mathcal{A}^{+}=$ $\left\{r M / r \in \mathbb{R}^{+}\right\}$where $M=\left(\begin{array}{rr}1 & 0 \\ 0 & -1\end{array}\right)$. Let $\varphi: \Gamma_{1} \rightarrow \Gamma_{2}$ be an isomorphism between non conjugate and non elementary Fuchsian groups. The groups $\Gamma_{1}$ and $\Gamma_{1 \varphi}$ are Zariski dense subgroups respectively of $G$ and $G^{\prime}$. Consider the isomorphism $\Psi: \Gamma_{1} \rightarrow \Gamma_{1 \varphi}$ defined by $\Psi(\gamma)=(\gamma, \varphi(\gamma))$. The limit cone of the graph group associated to $\Psi$ is included in $\left\{(r M, r M, s M) / r, s \in \mathbb{R}^{+}\right\} \subset \mathcal{A}^{+} \times \mathcal{A}^{+}$and hence has empty interior. According to Benoist's theorem (section 1), $\Gamma_{1 \Psi}$ is not Zariski dense. On the other hand $\Psi$ cannot be extended.

One deduces from the previous criterion the following corollary.

Corollary 2.2. Let Ad be the adjoint representation. If there exists an algebraic relation satisfied by all $(\operatorname{Ad}(\gamma)), \operatorname{Ad}(\varphi(\gamma))$ with $\gamma \in \Gamma$, then $\varphi$ can be extended to a continuous isomorphism between $G$ and $G^{\prime}$. 
In the case where $G=\operatorname{PSL}(n, \mathbb{R}), G^{\prime}=\operatorname{PSL}\left(n^{\prime}, \mathbb{R}\right)$ and $\varphi$ preserves the trace, Corollary 2.2 is proved in [16].

Remark that the condition $\ell(\gamma)=\ell(\varphi(\gamma))$ for each $\gamma \in \Gamma$ is not in general an algebraic condition. But in this case, since $\|v(\gamma)\|=\|v(\varphi(\gamma))\|$ for $\gamma \in \Gamma$, the limit cone of the graph group has empty interior. Applying Benoist's theorem, one concludes that $\Gamma_{\varphi}$ is not Zariski dense and hence that $\varphi$ can be extended. More generally, one has the following result.

Corollary 2.3. If the interior of $\mathcal{L}\left(\Gamma_{\varphi}\right)$ is empty then $\varphi$ can be extended to a continuous isomorphism between $G$ and $G^{\prime}$.

Let us give three different conditions under which $\Gamma_{\varphi}$ is not Zariski dense and hence $\varphi$ can be extended:

1) $\ell(\gamma)=\ell(\varphi(\gamma))$ for any $\gamma \in \Gamma$.

2) $v(\gamma)$ and $v(\varphi(\gamma))$ are colinear for any $\gamma \in \Gamma$.

3) The largest modulus of the complex eigenvalue or $\operatorname{Ad}(\gamma)$ equals the largest one of $\operatorname{Ad}(\varphi(\gamma))$ for any $\gamma \in \Gamma$.

Conditions 1) and 2) correspond to Theorems $\mathrm{A}$ and $\mathrm{B}$ when $G$ and $G^{\prime}$ are simple. Contrary to the conditions 1) and 2), if $\varphi$ satisfies condition 3) and $G$ and $G^{\prime}$ are not simple, $\varphi$ cannot be necessarily extended. For example, fix two isomorphic Schottky groups $\rho: \Gamma \rightarrow \Gamma^{\prime}$ in $\operatorname{PSL}(2, \mathbb{R})$. Suppose that $\ell(\gamma)>\ell(\rho(\gamma))$ for each $\gamma \in \Gamma$ (see [5] for the construction of such groups). Consider the isomorphism $\varphi: \Gamma \rightarrow \Gamma_{\rho}$ defined by $\varphi(\gamma)=(\gamma, \rho(\gamma))$. The groups $\Gamma, \Gamma_{\rho}$ are Zariski dense respectively in $\operatorname{PSL}(2, \mathbb{R})$ and $\operatorname{PSL}(2, \mathbb{R}) \times \operatorname{PSL}(2, \mathbb{R})$ and the condition 3$)$ is satisfied but $\varphi$ cannot be extended.

\section{Proofs of Theorems A and B}

In this section $G$ and $G^{\prime}$ denote connected semisimple groups with trivial center and without compact factor. Such a group can be decomposed into a product of connected noncompact simple groups with trivial center.

Lemma 3.1. Let $\Gamma, \Gamma^{\prime}$ be Zariski dense subgroups of $G$ and $G^{\prime}$. Suppose that $\varphi$ is a surjective homomorphism between $\Gamma$ and $\Gamma^{\prime}$ and set $\Gamma_{\varphi}=\{(\gamma, \varphi(\gamma)) / \gamma \in \Gamma\}$. The projections of the identity component of the Zariski closure of $\Gamma_{\varphi}$ into $G$ and $G^{\prime}$ are surjective.

Proof. The Lie algebra $\mathcal{G}$ of $G$ can be decomposed into a direct sum of simple ideals $\mathcal{G}=\mathcal{F}_{1}+\cdots+\mathcal{F}_{n}$. Moreover each ideal of $\mathcal{G}$ is a direct sum of certain $\mathcal{F}_{i}$ ([10] corollary II.6.3). Let $G_{i}$ be the connected Lie subgroup in $G$ associated to $\mathcal{F}_{i}$. Since $G$ has trivial center, $G=G_{1} \times \cdots \times G_{n}$. Let $H$ be the identity component of the Zariski closure of $\Gamma_{\varphi}$. Denote $p$ the projection of $H$ into $G$ and $T_{p}$ its tangent map at identity. The image, $\mathcal{F}$, of the Lie algebra of $H$ by $T p$ is a non trivial 
subalgebra of $\mathcal{G}$ normalized by $\Gamma$. Since $\Gamma$ is Zariski dense, $\mathcal{F}$ is an ideal and hence $\mathcal{F}=\mathcal{F}_{i_{1}}+\cdots+\mathcal{F}_{i_{k}}, k \leq n$. This implies that $p(H)=G_{i_{1}} \times \cdots \times G_{i_{k}}$. Since the index of $H$ in the Zariski closure of $\Gamma_{\varphi}$ is finite and $\Gamma$ is Zariski dense, $p(H)$ is also Zariski dense. This proves that $k=n$ and thus that $p$ is surjective. Since $\varphi$ is surjective, the same argument holds for the projection of $H$ into $G^{\prime}$.

Proof of Theorem A. Denote $H$ the identity component of the Zariski closure of $\Gamma_{\varphi}$ and $\mathcal{H}$ its Lie algebra. We want to prove that the projection $p$ (resp. $p^{\prime}$ ) of $H$ into $G$ (resp. $G^{\prime}$ ) is injective. Let us first show that $\mathcal{H}$ is semisimple. Consider its solvable radical $\mathcal{R} \subset \mathcal{H}$. The image of $\mathcal{R}$ by the tangent map $T p$ of $p$ at identity is normalized by $\Gamma$. Since $\Gamma$ is Zariski dense in $G, T p(\mathcal{R})$ is a solvable ideal. The semi simplicity of $G$ implies that $T p(\mathcal{R})$ is trivial. Since $\varphi$ is surjective, the same argument holds for $p^{\prime}$. This shows that $\mathcal{R}$ is trivial. Fix a Cartan decomposition $\mathcal{H}=\mathcal{P}^{\prime \prime}+\mathcal{T}^{\prime \prime}$ of $\mathcal{H}$, since $G \times G^{\prime}$ is semisimple, there exists a Cartan decomposition $\mathcal{P}+\mathcal{T}$ of the Lie algebra of $G \times G^{\prime}$ such that $\mathcal{P}^{\prime \prime} \subset \mathcal{P}$ and $\mathcal{T}^{\prime \prime} \subset \mathcal{T}$ ([10] VI exercise 8(i)). Choose a Weyl chamber $\mathcal{W} \subset \mathcal{P}^{\prime \prime}$ since $\mathcal{P}^{\prime \prime} \subset \mathcal{P}$ one has $\mathcal{W} \subset \mathcal{A} \times \mathcal{A}^{\prime}$ where $\mathcal{A}$ and $\mathcal{A}^{\prime}$ are Cartan subalgebras of the Lie algebra $\mathcal{G}, \mathcal{G}^{\prime}$ of $G$ and $G^{\prime}$. Let us analyze Ker $p$. This group is normalized by $\Gamma^{\prime}$ because $\varphi$ is surjective. Since $\Gamma^{\prime}$ is Zariski dense and the center of $G^{\prime}$ is trivial, either $\operatorname{Ker} p=\{\operatorname{Id}\}$ or $\operatorname{Ker} p$ is a normal non trivial Lie subgroup of $G^{\prime}$. In the last case, denote $\mathcal{I}$ the Lie algebra of the identity component of $\operatorname{Ker} p$. One has $\mathcal{I}=\mathcal{I}_{1}^{\prime}+\cdots+\mathcal{I}_{p}^{\prime}$ where $\mathcal{I}_{j}^{\prime}$ are noncompact simple ideals of $\mathcal{G}^{\prime}$ such that $\mathcal{G}=\mathcal{I}_{1}^{\prime}+\cdots+\mathcal{I}_{k}^{\prime}$ with $k \geq p([10]$ corollary II.6.3). It follows that $\mathcal{W}$ contains an element $a=(0, \omega) \in \mathcal{A} \times \mathcal{A}^{\prime}$ with $\|\omega\| \neq 0$. Since $\Gamma_{\varphi} \cap H$ is Zariski dense in $H$, according to Benoist's theorem, the interior of its limit cone, $\mathcal{L}^{\mathcal{W}}\left(\Gamma_{\varphi} \cap H\right)$, relatively to $\mathcal{W}$, is not empty. Moreover $\mathcal{L}^{\mathcal{W}}\left(\Gamma_{\varphi} \cap H\right)$ is included in $S=\left\{\left(u, u^{\prime}\right) \in \mathcal{A} \times \mathcal{A}^{\prime} /\|u\|=\left\|u^{\prime}\right\|\right\}$ because $\varphi$ preserves the translation length and $\mathcal{L}^{\mathcal{W}}\left(\Gamma_{\varphi} \cap H\right)$ is included in the image of the limit cone of $\Gamma_{\varphi} \cap H$ relatively to $\mathcal{A}^{+} \times \mathcal{A}^{\prime}+$ by the Weyl group. Let $b=\left(u, u^{\prime}\right)$ an element of the interior of $\mathcal{L}^{\mathcal{W}}\left(\Gamma_{\varphi} \cap H\right) \subset \mathcal{W}$. One can suppose $\|u\|=\left\|u^{\prime}\right\|=1$. Since the interior of $\mathcal{L}^{\mathcal{W}}\left(\Gamma_{\varphi} \cap H\right)$ in $\mathcal{W}$ is not empty, the intersection of the plane generated by $a$ and $b$ with $\mathcal{L}^{\mathcal{W}}\left(\Gamma_{\varphi} \cap H\right)$ contains an open disc. There is a contradiction with the fact that the intersection of this plane with $S$ is the curve $\left\{\alpha a+\beta b / 2 \alpha \beta\left\langle u^{\prime}, \omega\right\rangle+\alpha^{2}\|\omega\|^{2}=0\right\}$. In conclusion $p$ is injective. The same argument holds for $p^{\prime}$, because $\varphi$ is surjective. Applying the lemma 3.1, one obtains that $p$ and $p^{\prime}$ are bijective. Consider now the projections $q$ (resp. $q^{\prime}$ ) of the Zariski closure $\bar{\Gamma}_{\varphi}^{Z}$ of $\Gamma_{\varphi}$ into $G$ (resp. $G^{\prime}$ ). The maps $q$ and $q^{\prime}$ are surjective. Let us prove that they are injective. Take $g \in \operatorname{Ker} q$, for any $h \in H$ one has $q\left(g h g^{-1} h^{-1}\right)=$ Id. Since $H$ is normalized by $\bar{\Gamma}_{\varphi}^{z}$ and $p$ is injective, $g h=h g$. Using the fact that $p^{\prime}$ is surjective one obtains $p^{\prime}(g) g^{\prime}=g^{\prime} p^{\prime}(g)$ for any $g^{\prime} \in G^{\prime}$. Because the center of $G^{\prime}$ is trivial, $g=$ Id. The same argument also holds for $p^{\prime}$. Consider the map $f=p^{\prime} \circ p^{-1}$, it is a continuous isomorphism between $G$ and $G^{\prime}$ whose restriction to $\Gamma$ coincides with $\varphi$. 
Proof of Theorem B. The proof is similar to the previous one. Let us just adapt the end of the proof of Theorem A, when we suppose that Ker $p$ is nontrivial. Under this assumption one obtains that $\mathcal{W}$ contains an element $a=(0, \omega) \in \mathcal{A} \times \mathcal{A}$ with $\omega \neq 0$. Since $v(\gamma)=k(\gamma) v(\varphi(\gamma))$ for each $\gamma \in \Gamma$, the limit cone $\mathcal{L}^{\mathcal{A}^{+} \times \mathcal{A}^{+}}\left(\Gamma_{\varphi} \cap H\right)$ is included in $T=\left\{\left(u, u^{\prime}\right) \in \mathcal{A}^{+} \times \mathcal{A}^{+} / u\right.$ and $u^{\prime}$ are colinear $\}$ and hence $\mathcal{L}^{\mathcal{W}}\left(\Gamma_{\varphi} \cap H\right)$ is included in $\bigcup_{g \in \text { Weyl }} g T$ where Weyl is the Weyl group of $\mathcal{A} \times \mathcal{A}$. The interior of $\mathcal{L}^{\mathcal{W}}\left(\Gamma_{\varphi} \cap H\right)$ in $\mathcal{W}$ is not empty according to Benoist's theorem. It follows that for some $g \in$ Weyl, the interior $I$ of $g(T)$ is not empty in $\mathcal{W}$. Let $b=\left(u, u^{\prime}\right) \in I$. Since rank $G \geq 2$ one can assume that $u^{\prime}$ is not colinear to $w$. The intersection of the plane $P$ generated by a and $b$ with $I$ contains an open disc. There is a contradiction with the fact that the intersection of $T$ with $g^{-1}(P)$ is a line.

\section{Applications of Theorem A to the space of representations}

Fix a connected semisimple Lie group $G$ without compact factor and with trivial center, and a symmetric space $X$ associated to $G$. A subgroup of $G$ is said parabolic if it fix a point of the geometric boundary, $\partial X$, of $X$.

Proposition 4.1. Let $\Gamma$ be a nonparabolic subgroup of $G$ and $H$ the identity component of its identity component. If $H \neq G$ then $H$ fix a totally geodesic submanifold $Y \varsubsetneqq X$.

Proof. We thank P. Eberlein for helpful arguments.

The group $H$ is reductive or parabolic ([3] corollaire 3.3). The last case cannot happens because $H$ is normalized by $\Gamma$ which does not fix any point in $\partial X$. Let $H=S T$ be the Levi decomposition of $H$ where $S$ is a connected semisimple group and $T$ is a torus, corresponding to the identity component of the center of $H$. If $T \neq$ Id there exists a flat totally geodesic submanifold $T \subset X$ such that $T$ leaves $F$ invariant and $F / T$ is compact ([8]). Let $C$ be the union of all totally geodesic submanifolds which are parallel to $F$. Then $C$ is invariant under $H$ and is isometric to $F \times N$ for some closed convex subset $N$ of $X$ ([7] proposition 1.6.7). The set $C$ is a totally geodesic submanifold possible with boundary. Let $Y$ be a complete totally geodesic submanifold of $X$ with $\operatorname{dim} Y=\operatorname{dim} C$. Since $H$ leaves $C$ invariant and $C$ contains an open subset of $Y$, the group $H$ leaves $Y$ invariant. Remark that $Y \neq X$, because $Y$ contains an Euclidean factor. If $T=\{\operatorname{Id}\}$ then $H$ is semisimple, and there exists $x \in X$ such that $H x$ is a totally geodesic submanifold ([13] lemma 7.21). By the assumption $H \neq G$ hence $H x \neq X$.

Let $\Gamma$ be an abstract group and $\rho: \Gamma \rightarrow G$ be a faithful representation. One always supposes that the Zariski closure, $H_{\rho}$, of $\rho(\Gamma)$ is connected and that the representation $\rho$ is nonparabolic (i.e. $\rho(\Gamma)$ is nonparabolic). In this case $H_{\rho}$ is reductive (proof of proposition 4.1). Let $H_{\rho}=\mathrm{ST}$ be the Levi decomposition 
of $H_{\rho}$. The representation $\rho$ is noncompact if $S$ is a semisimple group without compact factor and with trivial center. Under this assumption $H_{\rho}$ stabilizes a totally geodesic submanifold of $X$ isometric to $N \times F$ where $N$ is a symmetric space on which $S$ acts transitively and $F$ is a flat on which $T$ acts by translation with compact quotient (proof of the proposition 4.1). Two faithful, nonparabolic and noncompact representations $\rho$ and $\rho^{\prime}$ of $\Gamma$ are equivalent if there exists a isometry $f$ between $N \times F$ and $N^{\prime} \times F^{\prime}$ such that $f \circ \rho(\gamma)=\rho^{\prime}(\gamma) \circ f$ for any $\gamma \in \Gamma$. If $F$ and $F^{\prime}$ are empty, then $\rho$ and $\rho^{\prime}$ are equivalent if and only if $\rho^{\prime} \circ \rho^{-1}$ can be extended to a continuous isomorphism between $S$ and $S^{\prime}$ ([7] proposition 3.9.11). Denote $\mathrm{R}_{\text {fnpnc }} / \sim$ the space of faithful nonparabolic,noncompact representations of $\Gamma$ into $G$, up to the equivalence relation. The following result is an application of Theorem A to the context of representations.

Proposition 4.2. The map $L: R_{f n p n c} / \sim \rightarrow \mathbb{R}^{\Gamma}$ defined by $L([\rho])(\gamma)=\ell(\rho(\gamma))$ is injective.

Proof. Let $\rho_{1}, \rho_{2} \in \mathrm{R}_{\text {fnpnc }}$. Suppose $L\left(\rho_{1}\right)=L\left(\rho_{2}\right)$. For $i=1,2$ set $\Gamma_{i}=$ $\rho_{i}(\Gamma), H_{i}=H \rho_{i}$ and $H_{i}=S_{i} T_{i}$.

a) Suppose $S_{1}=S_{2}=\{e\}$, then $T_{i}$ acts by translation on the flat $\left(F_{i},\langle\rangle_{i}\right)$ and $F_{i} / T_{i}$ is compact. Let us identify $\rho_{i}(\gamma)$ with its translation vector. Choose a basis, $\rho_{1}\left(\gamma_{1}\right), \cdots, \rho_{1}\left(\gamma_{n}\right)$ of $F_{1}$, such a basis exists because $\Gamma_{1}$ is Zariski dense in $T_{1}$. For $\gamma \in \Gamma$, write $\rho_{1}(\gamma)=\sum_{i=1}^{n} a_{i} \rho_{1}\left(\gamma_{i}\right)$ and $\rho_{2}(\gamma)=\sum_{i=1}^{n} b_{i} \rho_{2}\left(\gamma_{i}\right)+\omega$ where $\omega$ is orthogonal to each $\rho_{2}\left(\gamma_{i}\right)$. Since $\left\|\rho_{1}(\gamma)\right\|=\left\|\rho_{2}(\gamma)\right\|$, one has $\left\langle\rho_{1}(\gamma), \rho_{1}\left(\gamma^{\prime}\right)\right\rangle_{1}=$ $\left\langle\rho_{2}(\gamma), \rho_{2}\left(\gamma^{\prime}\right)\right\rangle_{2}$ for any $\gamma, \gamma^{\prime} \in \Gamma$. Put $c_{i j}=\left\langle\rho_{1}\left(\gamma_{i}\right), \rho_{1}\left(\gamma_{j}\right\rangle_{1}=\left\langle\rho_{2}\left(\gamma_{i}\right), \rho_{2}\left(\gamma_{j}\right)\right\rangle_{2}\right.$. One has $\left\langle\rho_{1}(\gamma), \rho_{1}\left(\gamma_{j}\right)\right\rangle_{1}=\sum_{i=1}^{n} a_{i} c_{i j}$ and $\left\langle\rho_{2}(\gamma), \rho_{2}\left(\gamma_{j}\right)\right\rangle_{1}=\sum_{i=1}^{n} b_{i} c_{i j}$ hence $\sum_{i=1}^{n}\left(a_{i}-b_{i}\right) c_{i j}=0$ for any $1 \leq j \leq n$. This proves that $a_{i}=b_{i}$. Moreover $\left\|\rho_{1}(\gamma)\right\|=\left\|\rho_{2}(\gamma)\right\|$ hence $\omega=0$. One thus obtains $\rho_{2}(\gamma)=\sum_{i=1}^{n} a_{i} \rho_{2}\left(\gamma_{i}\right)$ and $\operatorname{dim}$ $F_{2}=n$ because $\Gamma_{2}$ is Zariski dense in $T_{2}$. The linear map $f: F_{1} \rightarrow F_{2}$ defined by $f\left(\rho_{1}\left(\gamma_{i}\right)\right)=\rho_{2}\left(\gamma_{i}\right)$ is an isometry satisfying $f \circ \rho_{1}(\gamma)=\rho_{2}(\gamma) \circ f$, hence $\left[\rho_{1}\right]=\left[\rho_{2}\right]$.

b) Suppose $S_{1} \neq\{e\}$, then $S_{2} \neq\{e\}$. Decompose $S_{i}$ into a product of noncompact simple factors with trivial center $S_{i}=S_{i 1} \times \cdots \times S_{i k_{i}}$ and denote $p_{i s}$ the projection of $S_{i}$ into $S_{i s}$. Since $\Gamma_{i}$ is Zariski dense in $S_{i} \times T_{i}$ then $p_{i s}(\Gamma)$ is Zariski dense in $S_{i s}$. Set $D=[\Gamma, \Gamma]$ and $D_{i}=\rho_{i}(D)$. The group $D_{i}$ is normalized by $\Gamma_{i}$ and is included in $S_{i}$, hence one can suppose that the Zariski closure of $D_{i}$ equals $S_{i 1} \times \cdots \times S_{i n_{i}}$ with $n_{i} \leq k_{i}$. Moreover $n_{i}=k_{i}$ because $p_{i s}\left(D_{i}\right)$ is normalized by $p_{i s}(\Gamma)$ which is Zariski dense in $S_{i s}$ and the center of $S_{i s}$ is trivial. In conclusion $D_{i}$ is Zariski dense in $S_{i}$. By assumption $\ell\left(\rho_{1}(d)\right)=\ell\left(\rho_{2}(d)\right)$ for any $d \in D$. One deduces from Theorem A that the restriction of $\rho_{2} \circ \rho_{1}^{-1}$ to $D_{1}$ can be extended to a continuous isomorphism $\varphi$ between $S_{1}$ and $S_{2}$. Up to $\varphi$, one can suppose $S_{1}=S_{2}$ and $\rho_{1}(d)=\rho_{2}(d)$ for any $d \in D$. Let $\gamma \in \Gamma$, since $\rho_{1}\left(\gamma d \gamma^{-1}\right)=\rho_{2}\left(\gamma d \gamma^{-1}\right)$ and $\rho_{1}(d)=\rho_{2}(d)$, the projection of $\rho_{2}^{-1}(\gamma) \rho_{1}(\gamma)$ into $S_{1}$ commutes with all $\rho_{1}(d)$ Since $D_{1}$ is Zariski dense and the center of $S_{1}$ is trivial, the projection of $\rho_{2}^{-1}(\gamma) \rho_{1}(\gamma)$ into $S_{1}$ is trivial. Consider now the projection $p_{i}$ of 
$\Gamma_{i}$ into $T_{i}$. One has $\ell\left(p_{1} \circ \rho_{1}(\gamma)\right)=\ell\left(p_{2} \circ \rho_{2}(\gamma)\right)$, moreover $p_{i}\left(\Gamma_{i}\right)$ is Zariski dense in $T_{i}$. Using arguments developped in a), one obtains the existence of a isometry $f: F_{1} \rightarrow F_{2}$ such that $f \circ\left(p_{1} \circ \rho_{1}(\gamma)\right)=p_{2} \circ \rho_{2}(\gamma) \circ f$, hence $\left[\rho_{1}\right]=\left[\rho_{2}\right]$.

The following part is inspired by the section 5 of A. Parreau's thesis ([15]). Let us consider the particular case where $\Gamma$ is an infinite group of finite type. Fix a finite set, $S$, of generators. One associates to a representation $\rho: \Gamma \rightarrow G$ its minimal displacement, $\lambda(\rho)=\operatorname{Inf}_{x \in X}\left(\operatorname{Sup}_{s \in S} d(x, \rho(s)(x))\right.$. If $\lambda(\rho)=0$ there exists a sequence $\left(x_{n}\right)_{n \geq 1}$ in $X$ such that $\lim _{n} d\left(x_{n}, \rho(s)\left(x_{n}\right)\right)=0$ for any $s \in S$. Up to a subsequence one can suppose that $\left(x_{n}\right)_{n \geq 1}$ converges in $X \cup \partial X$. If $\lim _{n} x_{n}=x \in X$ then $\rho(s)(x)=x$ for any $s \in S$ and hence $\rho(\Gamma)$ belongs to a compact subgroup. Otherwise $\lim _{n} x_{n}=\xi \in \partial X$ and $\rho(s)(\xi)=\xi$ for any $s \in S$. In this case $\rho$ is parabolic. In conclusion, if $\rho \in \mathrm{R}_{\text {fnpnc }}$ then $\lambda(\rho)>0$. Let us consider the map $\frac{V}{\lambda}: \mathrm{R}_{f n p n c} / \sim \mathbb{R}^{\Gamma}$ defined by $L([\rho])(\gamma)=\frac{\ell(\rho(\gamma))}{\lambda(\rho)}$. This map is continuous $([15]$ propositions V.2.3 and V.3.8) and its image is included in a compact set ([15] proposition V.4.1). One deduces from these properties and from the proposition 4.2 the following result.

Corollary 4.3. The map $\frac{L}{\lambda}: R_{f n p n c} / \sim \rightarrow \mathbb{R}^{\Gamma}$ is injective, continuous and its image is included in a compact set.

Acknowledgments. The authors deeply thank Y. Benoist and P. Eberlein for numerous discussions. The second author wants to thank G. Besson for his hospitality and inspiring discussions during his visit to Institut Fourier.

\section{References}

[1] Y. Benoist, Propriétés asymptotiques des groupes linéaires, GAFA 7 (1997), 1-47.

[2] G. Besson, G. Courtois and S. Gallot, Entropies et rigidités des espaces localement symétriques de courbure strictement négative, GAFA $\mathbf{5}$ (1997), 731-799

[3] A. Borel and J. Tits, Eléments unipotents et sous-groupes paraboliques de groupes réductifs. Inv. math. 12 (1971), 95-104.

[4] M. Burger, Intersection, the Manhattan curve and Patterson-Sullivan theory in Rank 2, International Mathematics Research Notices 7 (1993), 217-225.

[5] F. Dal'Bo, Famille de groupes agissant sur le produit de deux variétés de Hadamard, Séminaire de théorie spectrale et géométrie de Grenoble (1997).

[6] F. Dal'Bo and I. Kim, A criterion of conjugacy for Zariski dense subgroups, CRAS t. 330, série I (2000), 647-650.

[7] P. Eberlein, Geometry of nonpositively curved manifolds, Univerisity of Chicago Press, 1996.

[8] D. Gromoll and J. Wolf, Some relations between the metric structure and the algebraic structure of the fundamental group in manifolds of nonpositive curvature, Bull. Amer. Math. Soc. 77 (1971), 545-552. 
[9] U. Hamenstadt, Cocycles, symplectic structures and intersection, GAFA 9 (1) (1999), 90140.

[10] S. Helgason, Differential geometry, Lie groups and symmetric spaces, Academic Press, 1978.

[11] I. Kim, Marked length rigidity of rank one symmetric spaces, Topology, $40 \mathrm{n}^{\circ} 6$ (2001), 1295-1323.

[12] I. Kim, Ergodic theory and rigidity on the symmetric space of noncompact type, Ergodic theory and dynamical systems, $21 \mathrm{n}^{\mathrm{O}} 1$ (2001), 93-114.

[13] G. Margulis, Discrete subgroups of semisimple Lie group, Springer-Verlag, 1991.

[14] J.-P. Otal, Le spectre marqué des surfaces à courbure négative, Ann. of Math. (2) 131 (1990), 151-162.

[15] A. Parreau, Dégénérescences de sous-groupes discrets de groupes de Lie semisimples et actions de groupes sur des espaces affines, Thèse, Orsay, 2000.

[16] J. H. Sampson, Sous-groupes conjugués d'un groupe linéaire, Ann. Inst. Fourier 26 (2) (1976), 1-6.

Françoise Dal'Bo

Université de Rennes 1

Institut de Recherche Mathématique de Rennes

Campus de Beaulieu

35042 Rennes cedex

France

e-mail: dalbo@univ-rennes1.fr

(Received: September 18, 2001)
Inkang KIM

Department of Mathematics

Seoul National University

151-741 Seoul

Korea

e-mail:inkang@math.snu.ac.kr

To access this journal online:

(40) http://www.birkhauser.ch 